Article

\title{
Online Education in Early Primary Years: Teachers' Practices and Experiences during the COVID-19 Pandemic
}

\author{
Kleopatra Nikolopoulou
}

Citation: Nikolopoulou, K. Online Education in Early Primary Years:

Teachers' Practices and Experiences during the COVID-19 Pandemic.

Educ. Sci. 2022, 12, 76. https:// doi.org/10.3390/educsci12020076

Academic Editor: João Piedade

Received: 19 December 2021

Accepted: 20 January 2022

Published: 21 January 2022

Publisher's Note: MDPI stays neutral with regard to jurisdictional claims in published maps and institutional affiliations.

Copyright: (C) 2022 by the author. Licensee MDPI, Basel, Switzerland. This article is an open access article distributed under the terms and conditions of the Creative Commons Attribution (CC BY) license (https:// creativecommons.org/licenses/by/ $4.0 /)$.
Department of Early Childhood Education, National and Kapodistrian University of Athens, 10680 Athens, Greece; klnikolop@ecd.uoa.gr

\begin{abstract}
During the COVID-19 pandemic, educational institutions around the world were forced to close and the academic process continued online. Online teaching can support students' learning, but little research exists in the foundational early primary years (children aged 4-8). The purpose of this study was to investigate teachers' practices and experiences in implementing online teaching during the pandemic period. The participants were 14 Greek teachers of early primary years, and data were collected via interviews. The findings indicated that most of the teachers used a combination of teaching practices-approaches, while the learning activities implemented mainly regarded language, psychomotor activities, display of videos, mathematics and interdisciplinary activities. Teachers' feelings were initially negative, while afterwards they experienced more positive feelings. Disadvantages of online education, as experienced by teachers, mainly regarded technical problems, followed by limited resources/support for children at home, and limited training in online methodology. Teachers' positive experiences regarded children's familiarization with the technology and maintenance of contact with the school environment, while the role of the parents was revealed as essential. Implications and suggestions for future online education in early primary years include maintenance of good high-standard teaching practices, enhancement of digital culture and parents involvement with children's activities at home.
\end{abstract}

Keywords: online education; early primary years; teacher practices; teacher experiences; COVID19 pandemic

\section{Introduction}

The COVID-19 outbreak was declared by the World Health Organization to be a global pandemic in March 2020 [1]. Educational institutions were forced to close in many countries worldwide, the educational process continued online [2], and the obligation of teachers to utilize digital technologies in education emerged [3]. Digital technology played an important role in enabling teachers to implement online education by using various digital platforms and tools [4]. Online teaching during the COVID pandemic was an unprecedented case, especially for early primary education; it is an unusual means of education for young children [5]. However, there is little empirical evidence in the foundational early primary years/grades during the COVID-19 pandemic (e.g., [5-7]), in comparison to studies in other sectors of education such as secondary schools or universities.

The purpose of this study was to investigate Greek teachers' practices and experiences when implementing online teaching in early primary years during the pandemic (teaching children aged 4-8). In Greece, similarly to many other countries, the emergency transition started in the spring of 2020 (during which in-person education transitioned to online teaching and learning) and ended in June 2021. This study is important because teacher practices affect young children's engagement-learning and contribute towards a more sustainable online education. Additionally, there is limited published research in the early primary years during the COVID-19 pandemic, while the role of early years' educators was highlighted as essential during this period [8]. For the purpose of this paper, as the 
age-boundaries between preschool and first year of primary school differ among different countries, the terms early childhood, preschool and early primary/elementary years are used as synonymous, indicating the formal educational settings that attend children aged between 4 and 8 years old. Also, the terms (digital) technology and ICT are used synonymously.

\section{Literature Review}

Yildirim [5], in Turkey, investigated how preschool education was implemented during the pandemic, what kind of activities were held, what challenges need to be overcome, and what measures need to be taken to sustain preschool education. Most teachers stated that the pandemic has negatively affected students (emotional, cognitive and psychomotor skills, teacher-student interaction), while some teachers reported higher parental engagement in their children's education. The activities performed were art, (Turkish) language, science, drama, music, mathematics, and games, while the skills-concepts they would like their students to develop included collaboration, flexibility, as well as learning hygiene, numbers, and shape-related concepts. Teachers reported they communicated with parents and students mostly on the phone, WhatsApp, followed by video-call, Facebook, or Instagram, while major challenges regarded internet connection issues, and reluctant parents. Similarly, in another study in Turkey [9] the activities most preferred by preschool teachers were games and art activities about hygiene, and communication with parents was over internet platforms (WhatsApp, zoom, skype) or telephone calls. Teachers expressed some negative feelings (e.g., anxiety, worry, fear), while a positive effect of the pandemic was parents' participation in children's educational activities (increase of parent-child interaction).

In the same country, Alan [10] identified the needs of early childhood teachers regarding online education during the pandemic. It was found that teachers need to improve their technological competencies, have more interactive resources and a user-friendly educational platform (to provide educational activities and facilitate communication), as well as have support for their psychological well-being (since some negative feelings such as anxiety were identified).

Hu et al. [7] provided evidence of how preschool teachers applied online resources (e.g., via digital-mediated learning platforms) for young children during COVID-19, in Hong Kong. Some barriers included difficulty engaging their children when online and inadequate support from parents for learning activities; teachers who perceived greater engagement of children or support from parents were more likely to expect online teaching in the future. The need for more interactive online teaching preparation was suggested.

Timmons et al. [6] examined the implementation and impact of online teaching in early primary contexts (kindergarten, grades 1 and 2) during the pandemic in Canada. The challenges perceived by teachers included equity issues (concerns with access to technology, support at home), social, emotional and academic effects of online teaching for children, as well as effects on parents. Recommendations about the improvement of online teaching-learning in the (post) COVID era were provided (encouraging synchronous and asynchronous teaching, individual instruction for students, etc).

Steed and Leech [11], in the USA, reported that the most utilized learning activities were singing songs and reading stories online. Teachers expressed concerns such as missing in person interactions with children, concerns about children with special needs, inadequate resources, and lack of guidance from administrators (about online education). The role of families was crucial since teachers relied on them for the implementation of children's activities at home.

Atiles et al. [12] explored the experiences of teachers of 3-6-year-old children in the USA and some Latin American countries, during the pandemic. The findings revealed that various platforms were used to communicate with parents, while half of the sample faced internet connectivity problems. Teachers expressed concerns for children's and families' mental health, they questioned the suitability of online learning for young children, and reported lack of preparation/training for online teaching. 
Accordingly, the current study was carried out in order to examine teachers' practices and experiences when implementing online teaching in early primary years, during the pandemic. In line with the purpose of the study (mentioned in Introduction), the following research questions $(\mathrm{RQ})$ were addressed:

RQ1. What were teachers' practices during the pandemic?

RQ2. What learning activities were implemented during the pandemic?

RQ3. What were teachers' feelings and experiences (positive and negative) in implementing online education?

RQ4. What was the role of the parents and children's experiences (as experienced by teachers)?

\section{Materials and Methods}

\subsection{Sample}

The sample of the study consisted of 14 teachers working in public settings, in North and South Greece; teachers were teaching children aged 4-8 years. In Greece, children aged 4-6 years attend preschool, while children aged 6-7 and 7-8 years attend the first and second year of primary/elementary school, respectively. Table 1 shows the demographic characteristics of the sample (gender, years of teaching experience, ages, years of ICT use in class for educational purposes, preparation to teach online courses). A total of 8 teachers teach in preschools and 6 teach in the first years of primary schools, while most of the participants had 1-10 years of teaching experience. Teachers' experience with online education ranged between $1-1.5$ years.

Table 1. Demographic information of teachers $(N=14)$.

\begin{tabular}{ccc}
\hline Gender & Years of teaching experience & Ages \\
\hline Female (13) & $1-10(12)$ & $25-30(10)$ \\
Male (1) & $20+(2)$ & $31-35(2)$ \\
& & $41+(2)$ \\
\hline Years of ICT use in class & Preparation to teach online courses & School level \\
\hline $1-4(10)$ & Informal on-the-job training (8) & Preschool (8) \\
$5-10(2)$ & None (6) & Primary (6) \\
$10+(2)$ & & \\
\hline
\end{tabular}

\subsection{Procedure and Research Instrument}

The data were collected via interviews with 14 teachers, between October and November 2021, i.e., immediately after educational institutions returned back to face-to-face education. Qualitative types of approaches are suitable and are often used in preschool/elementary settings [13]. Teachers' participation was voluntary and ethical issues were considered; all participants were assured that, should they wish to participate in the research, their comments and input would remain anonymous. Official permission was obtained from the University's ethics research committee.

The interview questions were guided by information emerging from international research, and were in line with this study's research questions. Indicative interview questions were as follows.

For the investigation of the first research question (RQ1): What teaching practices/approaches did you follow during online teaching? How was technology used with the students?

For the investigation of the second research question (RQ2): What learning activities were implemented during the pandemic?

For the investigation of the third research question (RQ3): What were your feelings when you implemented online teaching during the pandemic? What were your negative experiences in implementing online education (disadvantages of online education)? What were your positive experiences (advantages of online education)? 
For the investigation of the fourth research question (RQ4): What was the role of parents during online education? What kind of communication did you have with parents? What were your students' experiences during online education?

Additionally, information regarding teachers' characteristics (see Table 1) was collected via relevant questions. Each interview lasted for about 10-14 min. The interviews were conducted in a quiet place, at a time period that did not disturb the participants' teaching duties. Trustworthiness of the data was established by providing participants with opportunities to clarify meaning and intended points of discussion [14].

\subsection{Data Analysis}

Content-thematic analysis was used, and the codes for the data analysis were descriptive. Through the process of coding, patterns of responses were used to inform themes and categories generated in line with their relevance to the research questions [15]. Teachers' responses were thematically grouped into those which related to the pre-determined themes of teachers' practices and experiences. Anonymity was confirmed so as to eliminate possible untruthful responses. The results are presented according to the four research questions of the study; in excerpts, the codes T1-T14 were used for teachers (T1: Teacher 1, T2: Teacher 2, etc).

\section{Results}

In the presentation of results, in Tables $2-5$, the first column indicates teachers' responses and the second one indicates the number of references. It is noted that different responses may correspond to (derive from) the same teacher. For example, with regard to teaching practices a teacher may have reported traditional approaches as well as group activities; distinct key phrases/responses were coded as different categories.

Table 2. Teachers' practices during the pandemic $(N=14)$.

\begin{tabular}{|c|c|}
\hline \multicolumn{2}{|l|}{ What teaching practices/approaches did you follow during online learning? } \\
\hline $\begin{array}{l}\text { Combination of teaching approaches (e.g., constructivist \& behavioral approaches, } \\
\text { constructivism \& project work, online worksheets \& experiential activities) }\end{array}$ & 7 \\
\hline Traditional and behavioral approaches & 3 \\
\hline Experiential learning approaches (e.g., focus on activities behind the camera) & 2 \\
\hline Group activities & 2 \\
\hline Individual work (e.g., worksheets) & 2 \\
\hline Repetition of material in groups & 1 \\
\hline \multicolumn{2}{|l|}{ How was technology used with the students? } \\
\hline $\begin{array}{l}\text { Initially * with the help of the parents (parents had to also become familiar with } \\
\text { technology), then ** it was easier for children (and parents) to use the technology }\end{array}$ & 6 \\
\hline Some children used it easily, some had difficulties & 4 \\
\hline (mainly) Use of camera and microphone & 2 \\
\hline Use of several tools to design activities & 2 \\
\hline With difficulty (some children did not have resources) & 2 \\
\hline Then ${ }^{* *}$ the children were familiarized with the technology & 2 \\
\hline The possibilities for children to be active was not utilized & 1 \\
\hline \multicolumn{2}{|c|}{$\begin{array}{l}\text { Initially *: during the first year of online education (March 2020-May 2020). Then }{ }^{* *} \text { : during the second year o } \\
\text { online education (autumn 2020-spring 2021). }\end{array}$} \\
\hline \multicolumn{2}{|l|}{ Activities implemented } \\
\hline Worksheets, photocopies & 9 \\
\hline (Greek) Language activities (e.g., reading stories, writing) & 9 \\
\hline Psychomotor activities (to get up from the chair) & 6 \\
\hline Display of videos & 6 \\
\hline Mathematics activities & 5 \\
\hline Interdisciplinary activities (e.g., language and maths) & 5 \\
\hline
\end{tabular}


Table 3. Cont.

\begin{tabular}{cc}
\hline Activities implemented & 4 \\
Constructions with plasticine & 3 \\
Experiments (with parents' help) & 3 \\
Quiz (in synchronous mode) & 3 \\
Ready activities from photodentro platform (asynchronous) & 2 \\
photos via computer) & 1 \\
Project activities (continuation of face-to-face) & \\
Examples of activities in language & 5 \\
\hline Construction/creation of letters with plasticine & 5 \\
Writing in worksheets (write letters, etc.) & 3 \\
Group work (e.g., in Webex 'rooms' to write a paragraph) & 2 \\
Reading (e.g., in Webex 'rooms') & 2 \\
\hline Examples of activities in mathematics & \\
\hline Counting (e.g., to find and count objects that start with a specific letter) & 5 \\
Kinetic activities with numbers, enumeration (e.g., get up, turn, bring, show) & 4 \\
Counting and writing in worksheets & 3 \\
Construction/formation of numbers with simple materials & 2 \\
Activities with dates (e.g., digital calendar) & 2 \\
Concepts 'less than',' greater than' & 1 \\
\hline
\end{tabular}

Table 4. Teachers' feelings and experiences during the pandemic.

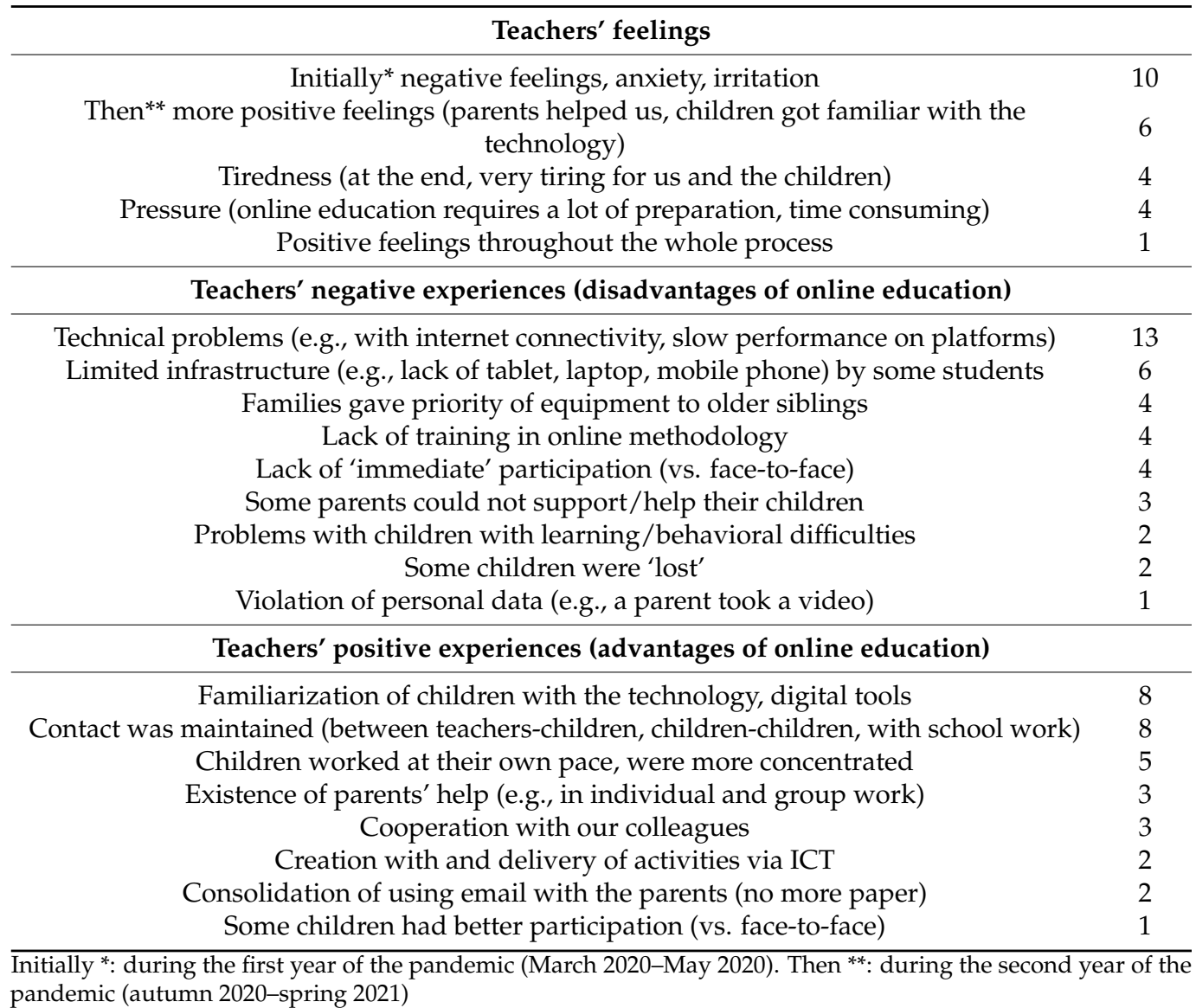
pandemic (autumn 2020-spring 2021) 
Table 5. Parents' role and children's experiences during the pandemic.

\begin{tabular}{|c|c|}
\hline \multicolumn{2}{|l|}{ Parents' role } \\
\hline Very important the parents' role, communication with parents & 9 \\
\hline Cooperation with parents, parents set the limits & 4 \\
\hline Some parents had to acquire digital competencies/skills & 4 \\
\hline $\begin{array}{c}\text { Initially * parents were frequently next to their children, then ** almost all children } \\
\text { were alone (in online education) }\end{array}$ & 3 \\
\hline Many parents were continually next to their child & 2 \\
\hline Parents had an active role (e.g., in finding materials needed) & 2 \\
\hline Some parents intervened with the teaching process & 2 \\
\hline \multicolumn{2}{|l|}{ Communication with parents } \\
\hline viber, messenger & 8 \\
\hline telephone & 8 \\
\hline email & 7 \\
\hline via the platform & 2 \\
\hline via blog & 1 \\
\hline \multicolumn{2}{|l|}{ Children's experiences } \\
\hline $\begin{array}{c}\text { Negative feelings (they missed physical contact, their friends, did not like online } \\
\text { education) }\end{array}$ & 10 \\
\hline Developed/exercised their digital skills, familiarization with the technology) & 8 \\
\hline $\begin{array}{c}\text { Initially* enthusiasm, greater participation, then }{ }^{* *} \text { children were tired, lower } \\
\text { participation }\end{array}$ & 4 \\
\hline Children liked psycho/music-motor activities & 3 \\
\hline Children got bored with traditional activities & 2 \\
\hline Children learned behavior rules (e.g., when to switch on/off the microphone) & 2 \\
\hline Very little participation (by some children) & 2 \\
\hline
\end{tabular}

Initially *: during the first year of the pandemic (March 2020-May 2020). Then **: during the second year of the pandemic (autumn 2020-spring 2021).

\subsection{Teachers' Practices during the Pandemic}

Table 2 shows teachers' practices during the pandemic. Half of the sample (7 teachers) reported a combination of teaching practices/approaches such as constructivist \& behavioral approaches, constructivism \& project work, online worksheets \& experiential activities. 3 references regarded traditional and behavioral approaches, followed by experiential learning approaches (e.g., focus on activities behind the camera). Regarding the facilitation of technology use, the teachers reported that initially (during the first year of online education) children used the technology with the help of their parents, while afterwards (second year of online education) it was easier for children (and parents) to use the technology (6 references). A few teachers mentioned that some children used the technology easily, while others faced difficulties (4 references). It is noted that, during online education, the platforms Webex (synchronous) and e-me (https: / /auth.e-me.edu.gr/, asynchronous educational platform, accessed on 15 December 2021), as well as the photodentro (http:/ / photodentro.edu.gr/, Greek National aggregator of educational content, accessed on 15 December 2021) were used.

Regarding teaching practices/approaches, several responses included a combination of teaching approaches, and indicative excerpts were:

"It was a combination of teaching approaches, strategies, etc. It had elements of behaviorism, constructivism. Essentially, depending on the lesson, if the children had questions, and (depending on) the content and type of activity, we used specific elements from learning theories." (T2)

"We tried to be a little bit constructive or (to apply) a little project work, as much as we could ... we were very dependent on the parents, so as long as the parents cooperated, a small project came out." (T3) 
With regard to experiential approaches, one teacher noted "We tried to focus on experiential approaches. That is, things that children would either do behind the camera or find in their room/home and interact with them." (T7)

With regard to the way technology was used, some participants noted a difference between the first and the second year (lockdown periods) of the pandemic, and an example of response was: "In the first year, it was difficult for the parents, first of all because they had to learn the platforms, to develop digital skills ..., the toddlers, those who participated, adapted quickly and learned the conveniences provided by the platform, discovered let's say emoji ... The second year was much easier for the children, and the parents were more familiar (with the technology)." (T10)

The limited or lack of resources/infrastructure at children's homes also affected children's participation in online education. For example, one teacher mentioned "The use of technology with students was with great difficulty, because we had the problem that children did not have the means/resources." (T1)

\subsection{Learning Activities Implemented during the Pandemic}

Table 3 indicates the learning activities implemented during the pandemic. These mainly included worksheets-photocopies (9 references), Greek language activities (9 references), psychomotor activities (such as the children to get up from the chair), display of videos, mathematics and interdisciplinary activities. Examples in language, included activities with phonemes (e.g., find objects that start with a specific letter-phoneme), reading stories, and writing (e.g., in worksheets), while examples of activities in mathematics regarded counting and kinetic/psychomotor activities with numbers (e.g., get up, turn, bring, show).

One teacher described the activities in parallel to those they tend to do in the classroom (i.e., in face-to-face education). An example of a relevant excerpt was:

"For example, an activity in language was to find things inside their house that start with the phoneme sigma (S). Or, to design the letter $S$ in a worksheet and build it from above with a material (construction) ... To tell us words that start with sigma and try to make it all together, with open microphones, so imagine what was happening, a story. Things, that in essence we would do in the classroom." (T14)

Some participants expressed concerns about the synchronous mode of online education, claiming it was easier to work in asynchronous mode. For example, "in photodentro (platform) that has some games there, but this in asynchronous mode..., in synchronous mode it was more difficult ... We did more language activities, reading stories, discussions, also maths, psychomotor activities, as much as anyone could, safely at home, a little dance as well." (T3)

Other statements regarding the learning activities implemented were:

"I was uploading some videos on e-me (asynchronous platform) for the children to watch, but again there was a problem; some parents wanted me to send them, for example, the exercises through other media, such as viber and messenger, as they could not access e-me." (T8)

"In synchronous mode, we sent a quiz which we would do at the same time, but still, we were trying to do group activities, to divide them into 'rooms' and tell them you will write a short report or a paragraph, with ideas that you will all have together." (T9)

\subsection{Teachers' Feelings and Experiences during the Pandemic}

Table 4 shows teachers' feelings and experiences (both negative and positive) during the pandemic. Most of the teachers reported that initially (during the first year of the pandemic - online education) they had negative feelings, they felt anxiety, and irritation (10 references), while more positive feelings were experienced during the second year of online education. Regarding teachers' negative experiences (disadvantages of online education as experienced by teachers), almost all reported technical problems (13 references), followed by students' limited infrastructure (6 references) and priority of families' providing resources/equipment to older siblings (4 references). Regarding teachers' positive 
experiences (advantages of online education as experienced by teachers), they mainly reported familiarization of children with the technology and maintenance of contact (between teachers-children, children-children, with school work) (8 references, respectively).

Teachers were also asked about the support they had during the pandemic and their suggestions for the improvement of online education. Half of the sample (7 out of 14 teachers) mentioned that during the first year of online education there was minimal/no support; however, afterwards (during the second year) there were some seminars (e.g., support teams) and/or rapid in-service training from official bodies (e.g., Universities, Ministry of Education). Teachers' suggestions included provision of organized in-service training ( 8 out of 14 ), solving technical problems with the platforms (6 out of 14), provision of suitable material/activities for online education, and equity issues for all students.

Negative feelings were frequently mentioned, in particular, at the beginning of online education. Learning how to use new tools in a short time was viewed as stressful. For example, "Initially, when it all started, stress and anxiety. Because, we did not know exactly what to do, we did not know how to do it ... certainly annoyed when we saw that the platforms had problems, it (the whole process) was too slow." (T11)

Parents' support and the cognitive improvement of children affected teachers' feelings so as to become more positive. As one teacher reported: "In the beginning, I was very negative, because I say: -Oh, they (children) are in front of a computer, how are we going to grasp their interest? Keep them doing things ... Later, because we also had the help from the parents, I started to like it, because I saw that the children had also a cognitive improvement." (T12)

Teachers shared some disadvantages of online education (as experienced during the pandemic) such as loss of the immediacy of the lesson and limited infrastructure; in particular, limited infrastructure at home caused the children to fall behind. Examples of extracts associated with negative experiences were:

"The immediacy of the lesson was lost, that is, it was not so lively ... many parents could not support their children because they were working ... we did not receive timely training ... in the first phase (of online education) we were completely alone." (T4)

"At some homes, there was a problem with infrastructure, they did not have the necessary devices, or the number of devices to connect the children (with online lessons). Imagine a family with three children, the one who went to preschool did not participate in the lesson ... these little ones were tired from the mobile phone, they could not watch from the phone." (T10)

Some teachers explained that children's familiarization with the technology was indeed a positive experience from online education, and an example was: "I think the most important thing is that the children familiarized themselves with the whole process, with online learning ... they learned different digital tools, each child was given the opportunity to work at his own pace." (T8)

Another teacher mentioned that online education was helpful in order to think of different types of activities and cooperate with colleagues: "It helped me to think of activities that can be done in another way, can be done from/via the computer ..., it helped me to cooperate with the kindergarten teacher of the other class." (T2)

\subsection{The Role of the Parents and Children's Experiences during the Pandemic}

Table 5 indicates teachers' experiences with regard to the role of the parents and children's experiences, during the pandemic. The role of the parents and the communication with them were experienced/considered as very important by most of the teachers ( 9 references). The support of parents to their children was also pointed out and, in particular, during the first year of the pandemic (when parents and children were less familiar with the process of online education). With regard to children's experiences, the majority of the teachers (10 out of 14) reported that children had overall negative feelings (they missed physical contact, their friends, they did not like online education). Teachers reported that children developed/exercised their digital skills (8 references), and that at the beginning 
of the pandemic there was greater enthusiasm and participation (in comparison to the tiredness experienced by children towards the last months of online education).

Teacher responses revealed that the role of the parents and the communication with them during the lockdown periods was very important. Examples of responses, were:

"(we had) Continuous communication, very frequent, especially in the first days, when the parents were not familiar with the technology. In the early days we were constantly over a phone on viber. My communication with the parents was done mainly by phone, mainly through a viber group that I had set up." (T1)

"The role of the parents was also very important, because it is different from being in the classroom and managing eighteen children... They will listen to you much more easily. At a distance, however, it is not so manageable. So, the parents should set the limits (similarly, as these are) set by the teacher in the classroom. So, I think, it is essential that we cooperated (with parents)." (T14)

"It (parents' role) was energetic in finding materials we would need ... Some other parents were next to their children and sometimes they intervened ... (directing them) say this, say that." (T7)

With regard to children's experiences, most respondents expressed the belief that children had negative feelings and did not like online education. Indicative excerpts were:

"Generally, the children were tired, the time (lunch time 2-4 pm) was very inappropriate, sometimes they were bored ... their feelings were not positive. They liked some things. When we did music-kinetic things and when we played games like pantomime, games with finding a material, but, in general, they did not like it (online education)." (T5)

"I think they (children) did not like distance education. None of the kids was happy with webex (platform). That is, when we spoke, they wanted their school to open ... There was not as much involvement, as if they were lost. If I had not intervened to speak, the involvement would have been very little/low ... with the technology, they were quite familiar." (T6)

\section{Discussion and Conclusions}

\subsection{Discussion of Findings}

The findings of this study add evidence to the emerging literature on teacher practicesexperiences in the context of early primary education during the pandemic; a field with relatively little research evidence. The interviews with the teachers revealed some issues difficult to be explored through large-scale quantitative surveys, and this study contributes data into teachers' practices-experiences during the pandemic. Teacher narratives inform the findings and represent teachers' voices for the situation in pandemic.

The findings of this study revealed that, during online education, most of the teachers used a combination of teaching practices-approaches (constructivist, traditional and behavioral approaches, as well as experiential learning approaches), while the learning activities implemented mainly included language, psychomotor activities, display of videos, mathematics and interdisciplinary activities. It is interesting that despite the distance, teachers applied kinesthetic activities (experiential and hands on home activities) to reach their students and attract their interest. There is some agreement with earlier research in early primary years which reported similar activities; e.g., Yildirim [5] reported implementation of language and mathematics activities, while Steed and Leech [11] reported reading stories online. Regarding the way technology was used, the teachers reported that initially (during the first year of lockdown) children tended to use the technology with the help of their parents, while afterwards (subsequent lockdowns) it was easier to use the technology, while a few children faced difficulties.

Teachers' feelings were initially negative, while afterwards they experienced more positive feelings. This is in line with earlier studies $[9,10]$ which reported negative feelings and, in particular, at the beginning of the pandemic. Negative feelings and anxiety are 
obviously linked to the lack of earlier experiences (online education was a new situation for all teachers) and the lack of support (mainly, at the beginning of the pandemic). Disadvantages of online education, as experienced by teachers, mainly included technical problems, followed by limited resources/support for children at home, and limited teacher training in online methodology. Similar obstacles were reported by earlier research: e.g., internet connectivity problems [5,12], children's access to technology and/or support at home [6,7], and lack of preparation/training for online teaching [12]. When using technology-based infrastructure, equity issues for socioeconomically disadvantaged students and teachers need to be considered. Teachers' positive experiences (advantages of online education) mainly included children's familiarization with the technology and maintenance of contact with the school environment. Digital natives are familiarized (or get easily familiarized) with the digital technology/tools, and this has implications for the cultivation and enhancement of a digital culture in formal educational settings.

Teachers' experiences of the role of the parents, highlighted the importance of the parental role and of communication with parents during the pandemic. This finding is in agreement with earlier research which indicated the crucial role of families [11] and the involvement of parents in children's educational activities [5,9]. The communication with parents took place predominantly via telephone calls/viber/messenger, rather than over internet platforms (since the parents had also to familiarize themselves with the digital platforms). This study revealed that some parents became teachers at home to assist their children; it was crucial to maintain communication so children did not lose out on the planned activities.

Teachers also reported that the majority of their students had negative feelings during the online education (e.g., they missed physical contact, their friends), while they also exercised/developed their digital skills; the latter one was reported among the advantages of online education. Timmons et al. [6] also reported on social and emotional effects of the pandemic on young children.

In parallel, the findings of this study revealed some differences between the first and the second year/wave of online education, with regard to: (a) the way technology was used by children (e.g., initially with parents' help, then it was easier for the children); (b) teachers' feelings (initially more negative feelings and anxiety, afterwards more positive feelings); (c) parents' role (during the first year they were more frequently next to their children); (d) children's experiences (initially, enthusiasm and greater participation in comparison to the second year); (e) teacher support (during the second year there was some support via rapid seminars, group support).

\subsection{Conclusions-Suggestions}

Taking into account the findings of this study, some implications and suggestions for (future) online education in the foundational early primary years include maintenance of good-high-standard teaching practices, enhancement of digital culture and parents' involvement with children's activities at home. Initially, it is important to maintain good teaching practices/approaches, and not to diminish the standard of learning activities with the shift to online education. Good practices are expected to attract children's interest in getting involved with the activities designed by teachers. For example, the learning activities could include digital activities as well as kinesthetic activities; i.e., implementation of kinetic/psychomotor activities that attract children's interest and engagement. UNESCO [16] mentions that the pandemic is an opportunity to rethink curricula, as well as the educational process. By rethinking curricula (e.g., more creative, innovative, and flexible ones), educators can increase possibilities of successfully supporting young children's social outcomes [17]. Preschool curricula could incorporate guidelines for online activities. In parallel, enhancement of digital culture, is suggested to include equity issues, to ensure all children's access to technology at home; reducing inequalities is included in the sustainable development agenda of the United Nations [18]. Stakeholders (policy makers, curriculum developers, school principals) need to support a digital learning culture 
and teachers in the event that teaching-learning is shifted online in the future. For example, professional development initiatives could incorporate good practices for online teaching, practices utilizing digital tools, so as to enhance teachers' digital competencies and confidence (thus, eliminating feelings of anxiety). Policy makers are suggested to adopt a continuous improvement plan with good practices on confronting emergency crises. School principals could also devise emergency action plans for future crises situations. During the pandemic period, the role of school leaders/principals is important, since they contribute to the establishment of a digital learning culture in their schools [3]. The role of the parents is suggested to be empowered, via parents' support of children's educational activities, and communication and co-operation with teachers. Parents' digital skills will also prove useful for supporting their children and communicating with teachers.

\subsection{Limitations-Future Research}

The limitations of this study include the small sample size and its origin from one country. Future research is suggested to include a larger sample, and examine school characteristics (e.g., school context and leadership) in relation to teachers' practices. Future research could investigate ways of utilizing digital technology to eliminate barriers [19], and examine how to deal with various emergency situations in the early primary educational sector. Future studies could also explore digital pedagogy transition among different modes of online teaching and learning, and school development for crises situations [20].

Funding: This research received no external funding.

Institutional Review Board Statement: The study was conducted according to the guidelines of the Declaration of Helsinki, and approved by the Ethics Committee of Department of Early Childhood Education, National and Kapodistrian University of Athens, Greece.

Informed Consent Statement: ]Informed consent was obtained from all subjects involved in the study. Data Availability Statement: Not applicable.

Acknowledgments: The author would like to thank the teachers who participated voluntarily in this study.

Conflicts of Interest: The author declares no conflict of interest.

\section{References}

1. Cucinotta, D.; Vanelli, M. WHO Declares Covid-19 a Pandemic. Acta Biomed. Atenei Parm. 2020, 91, $157-160$.

2. UNESCO. 10 Recommendations to Ensure that Learning Remains Uninterrupted. 2020. Available online: https:/ / en.unesco.org/ news / covid-19-10-recommendations-plan-distance-learningsolutions (accessed on 11 October 2021).

3. Karakose, T.; Polat, H.; Papadakis, S. Examining Teachers' Perspectives on School Principals' Digital Leadership Roles and Technology Capabilities during the COVID-19 Pandemic. Sustainability 2021, 13, 13448. [CrossRef]

4. Starkey, L.; Shonfeld, M.; Prestridge, S.; Cervera, M.G. Special issue: Covid-19 and the role of technology and pedagogy on school education during a pandemic. Technol. Pedagog. Educ. 2021, 30,1-5. [CrossRef]

5. Yıldırım, B. Preschool education in Turkey during the Covid-19 pandemic: A phenomenological study. Early Child. Educ. J. 2021, 49, 947-963. [CrossRef] [PubMed]

6. Timmons, K.; Cooper, A.; Bozek, E.; Braund, H. The Impacts of COVID-19 on early childhood education: Capturing the unique challenges associated with remote teaching and learning in K-2. Early Child. Educ. J. 2021, 49, 887-901. [CrossRef] [PubMed]

7. Hu, X.; Chiu, M.M.; Leung, W.M.V.; Yelland, N. Technology integration for young children during COVID-19: Towards future online teaching. Br. J. Educ. Technol. 2021, 52, 1513-1537. [CrossRef] [PubMed]

8. Murray, J. In a time of COVID-19 and beyond, the World needs early childhood educators. Int. J. Early Years Educ. 2020, 28, 299-302. [CrossRef]

9. Duran, M. The effects of COVID-19 pandemic on preschool education. Int. J. Educ. Methodol. 2021, 7, 249-260. [CrossRef]

10. Alan, Ü. Distance education during the COVID-19 pandemic in Turkey: Identifying the needs of early childhood educators. Early Child. Educ. J. 2021, 49, 987-994. [CrossRef]

11. Steed, E.A.; Leech, N. Shifting to Remote Learning during COVID-19: Differences for Early Childhood and Early Childhood Special Education Teachers. Early Child. Educ. J. 2021, 49, 789-798. [CrossRef]

12. Atiles, J.T.; Almodóvar, M.; Chavarría Vargas, A.; Dias, M.J.A.; Zúñiga León, I.M. International responses to COVID-19: Challenges faced by early childhood professionals. Eur. Early Child. Educ. Res. J. 2021, 29, 66-78. [CrossRef] 
13. Nikolopoulou, K. Methods for investigating young children's learning and development with information technology. In Researching IT in Education: Theory, Practice and Future Directions; McDougall, A., Murnane, J., Jones, A., Reynolds, N., Eds.; Routledge: London, UK, 2010; pp. 183-191.

14. Creswell, J.W.; Creswell, J.D. Research Design: Qualitative and Quantitative Mixed Methods Approaches, 5th ed.; Sage Publications: London, UK, 2018.

15. Creswell, J.W. Educational Research: Planning, Conducting, and Evaluating Quantitative and Qualitative Research, 4th ed.; Pearson: Boston, MA, USA, 2012.

16. UNESCO. UNESCO Covid-19 Education Response: Education Sector Issue Notes. 2020. Available online: https://en.unesco.org/ covid19/educationresponse/issuenotes (accessed on 11 October 2021).

17. Spiteri, J. Quality early childhood education for all and the Covid-19 crisis: A viewpoint. Prospects 2021, 51, 143-148. [CrossRef]

18. United Nations. The Sustainable Development Agenda. 2020; United Nations: New York, NY, USA, 2020. Available online: https:/ / www.un.org/sustainabledevelopment/development-agenda/ (accessed on 19 November 2021).

19. Nikolopoulou, K. Mobile devices in early childhood education: Teachers' views on benefits and barriers. Educ. Inf. Technol. 2021, 26, 3279-3292. [CrossRef]

20. Bond, M. Schools and emergency remote education during the Covid-19 pandemic: A living rapid systematic review. Asian J. Distance Educ. 2020, 15, 191-247. 